\title{
HYBRID NUMERICAL-ANALYTICAL SCHEME FOR LOCALLY INHOMOGENEOUS ELASTIC WAVEGUIDES
}

\section{EVGENY V. GLUSHKOV ${ }^{1}$, NATALIA V. GLUSHKOVA ${ }^{1}$ AND ALEXANDER A. EVDOKIMOV ${ }^{1}$}

\author{
${ }^{1}$ Institute for Mathematics, Mechanics and Informatics, Kuban State University \\ Russian Federation, Krasnodar, 350040 \\ evg@math.kubsu.ru, evdokimovmail27@gmail.com
}

Key words: Guided Waves, Arbitrary Local Scatterers, Explicit Analytic Representations, Finite Element Software, Local-Global Solution

\begin{abstract}
Numerical simulation of guided wave excitation, propagation, and diffraction in laminate structures with local inhomogeneities (obstacles) is associated with high computational cost due to the need for a mesh-based approximation of extended domains with a rigorous account for the radiation conditions at infinity. To obtain computationally efficient solutions, hybrid numerical-analytical approaches are currently being developed, based on linking a numerical solution in a local vicinity of the source and/or obstacles with an explicit analytical representation in the external semi-infinite domain. However, the developed methods are generally not widely spread because the possibility of such coupling with an external multimode wave field is generally not provided in standard finite-element (FE) software. We propose a scheme that allows the use of the FE software as a black box for the required correct matching of local numerical and global analytical solutions (FEM-An). The FEM is used to obtain a set of local numerical solutions that serve as a basis in the inner domain. These solutions satisfy the boundary conditions induced by guided wave modes so that they fit correctly with the modal expansion in the outer region. The expansion coefficients of both FE and modal decompositions are determined then from the condition of stress and displacement continuity at the interface between the inner and outer domains. This scheme was numerically validated against analytical solutions to test problems and FE solutions for long waveguide sections with perfect match layer absorbing conditions at the ends (FEM - PML). Along the way, it turned out that the FEM-PML approach gives an incorrect result in the backward-wave bands and at high frequencies. The application of the FEM-An hybrid scheme is illustrated by examples of Lamb wave diffraction by elastic inclusions and delaminations.
\end{abstract}

\section{INTRODUCTION}

The relevance of numerical investigation of guided wave (GW) diffraction by local inhomogeneities (obstacles) is determined by numerous applications in such fields as mechanical engineering, non-destructive structural health monitoring (SHM), physical acoustics, geophysics, and others. Numerical simulation is often performed using standard (commercial) software that implements the finite element method (FEM). Such software is considered a versatile computer modeling tool because of its applicability to objects of various shapes, sizes, and materials and provided with a user-friendly interface, mesh generators, and 
post-processing facilities. However, its use in solving wave problems in extended domains (waveguides) encounters specific difficulties. They associate with a rapid increase in computational costs with an increase in the number of elements required for a good approximation of the oscillating wave field and the need to satisfy the radiation conditions at infinity.

Moreover, standard FEM software applies only to a finite domain. Its use with an infinite waveguide requires prior artificial limitation of the mesh area. For this, different types of absorbing or nonreflecting boundary conditions are set at the artificial boundaries, intended to correctly take into account the outflow of wave energy to infinity [1]. Among them, conditions of a perfectly matched layer (PML), which are used in much commercial software, have become especially popular [2].

In general, this approach works well, but with the steady-state time-harmonic oscillations, many superfluous spectral points of the corresponding boundary value problem appear in the frequency complex plane. Such points are inherent in restricted domains, but they are absent with the initial infinite guide. And the larger the limited domain is, the denser these additional unnecessary spectral points lie. At frequencies close to such spectral points, the FEM-PML solution can significantly differ from the desired solution for the infinite region. Besides that, the domain limited by the absorbing boundary conditions can include not only local inhomogeneity (scatterers), where the FEM use is essential, but also the source and measuring points. Therefore, the computational costs can still be too large because of the increasing number of mesh nodes needed to approximate the solution in both the diffraction zone and the entire wave propagation path from the source to the obstacles and sensors.

To reduce the cost, one can perform a mesh FE approximation only in local areas containing inhomo-

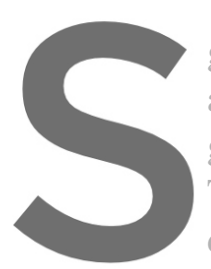

geneity and use explicit

as between the source

gives additional equations

The coupling can be ca

directly into the FEM
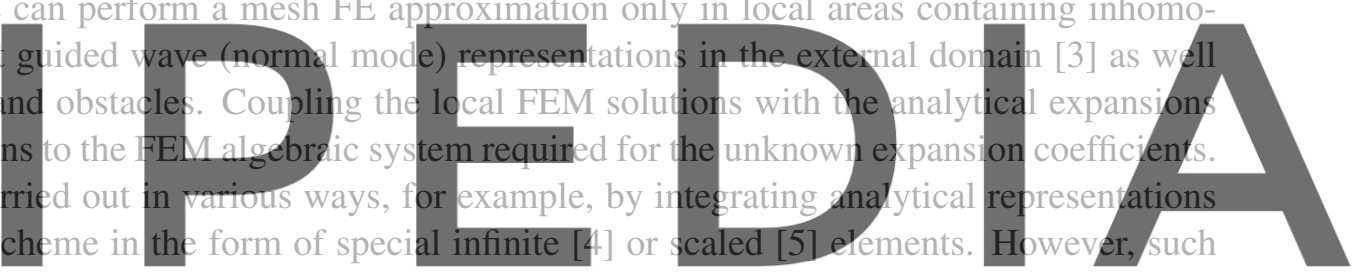

a method seems hardly possible with commercial FEM software, a "black box" for users who cannot

Register for freess the at https//www.scipedia.com to download the version without the watermark Nevertheless, we have developed a hybrid numerical-analytical scheme that uses standard FEM software only at the scatterers [6]. This scheme is based on the prior calculation of FEM solutions in the local subdomain with the boundary conditions induced by normal modes supported by the outer part of the waveguide. These FEM solutions are then used as a basis to match with the expansion in terms of guided waves propagating in the external domain. This GW expansion can be considered as a multimode infinite element that enables correct accounting for the wave energy outflow to infinity without introducing artificial absorbing boundary conditions. In this paper, we briefly repeat the scheme's description (Section 2) with the examples of its analytical and numerical verification and illustrate it with some numerical examples for elastic waveguides with multiple inclusions and layered structures with interface delaminations (Section 3). 


\section{FEM-ANALYTIC HYBRID SCHEME}

\subsection{Mathematical framework}

To give an idea of the local-global numeric-analytic approach (FEM-An), let us consider an isotropic layered elastic waveguide $D:|x|<\infty,|y|<\infty,-H<z<0$, with a local area $D_{\text {in }}:|x|<a$ containing different types of inhomogeneities (cracks, voids, inclusions, surface irregularities, etc., Fig.1). The geometry and material properties are independent of $y: \mathbf{u}=\left(u_{x}, u_{z}\right)=(u, w)$ and $\mathbf{x}=(x, z)$ (2D statement). The steady-state time-harmonic oscillations $\mathbf{u}(\mathbf{x}, \omega) e^{-i \omega t}$ can be caused by some source (e.g., surface load or volume force) located in $D_{i n}$ or by an incident guided wave $\mathbf{u}_{0}(\mathbf{x}) e^{-i \omega t}=\mathbf{a}_{0}(z) e^{i\left(\zeta_{0} x-\omega t\right)}$ coming from a remote source or infinity.

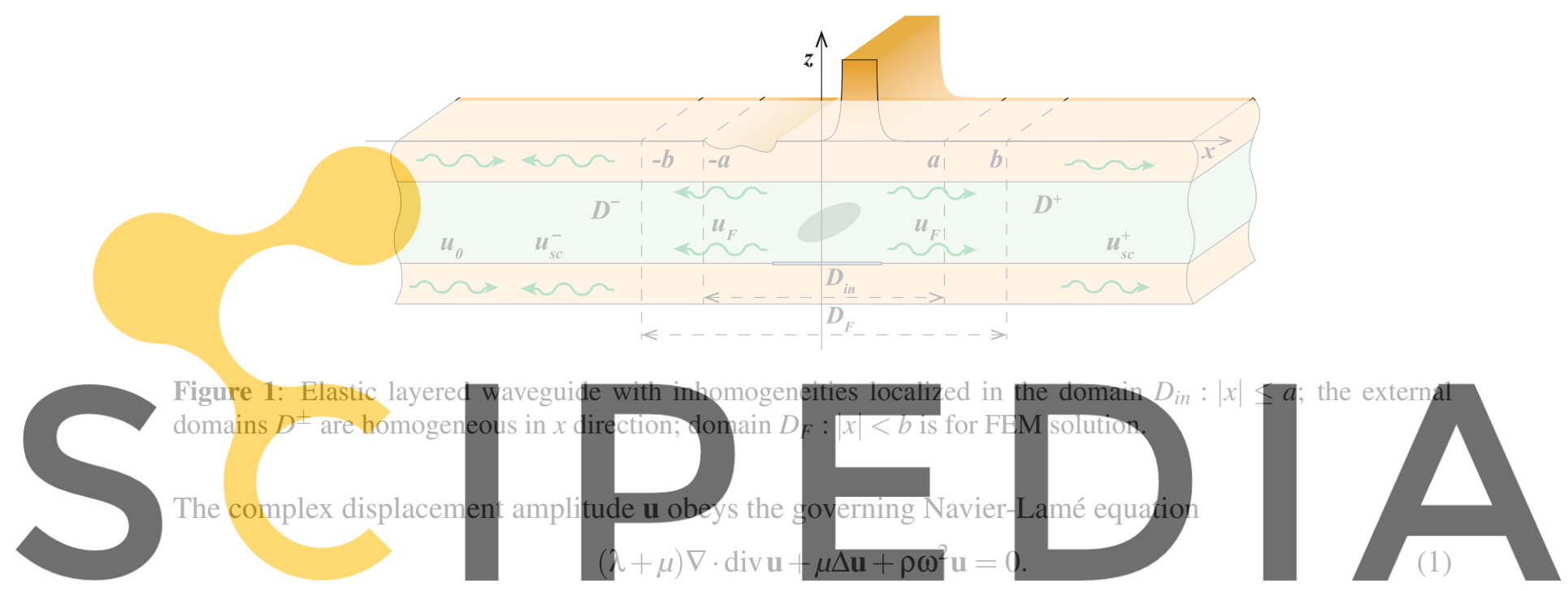

Register for free at https//Www.scipedia.com to download the version without the the watermark satisfies the conditions of stress and displacement continuity at the interfaces $z=z_{m} ; M$ is the number of sublayers. The outer sides $z=0$ and $z=-H$ are stress-free except a possible local area of external loading. The Lamé constants $\lambda$ and $\mu$, and the density $\rho$ are piece-wise constant functions of the transversal variable $z$.

The external domains $D^{-}: x<-a$, and $D^{+}: x>a$, do not contain scatterers. Therefore, the wave field $\mathbf{u}_{s c}$ scattered in the domain $D_{i n}$ can be represent here in terms of travelling and evanescent guided waves (normal modes):

$$
\mathbf{u}_{\mathrm{sc}}(x, z)=\sum_{n=1}^{\infty}\left\{\begin{array}{cl}
c_{n}^{-} \mathbf{a}_{n}^{-}(z) e^{-i \zeta_{n}(x+a)}, & x<-a \\
c_{n}^{+} \mathbf{a}_{n}^{+}(z) e^{i \zeta_{n}(x-a)}, & x>a
\end{array}\right.
$$

Here $c_{n}^{ \pm}$are unknown expansion coefficients, $\mathbf{a}_{n}^{ \pm}(z)$ are normal-mode eigenforms, and $\zeta_{n}$ are real and complex wavenumbers. Several first wavenumbers are purely real $\left(\operatorname{Im} \zeta_{n}=0, n=1,2, \ldots, N_{r}\right)$, they yield traveling guided waves. The others are complex ones arranged in ascending order of the imaginary parts $\left(\operatorname{Im} \zeta_{n+1} \geq \operatorname{Im} \zeta_{n}, n>N_{r}\right)$. They yield evanescent modes that exponentially decay with the distance:

$$
\left|\mathbf{a}_{n}^{ \pm} e^{ \pm i \zeta_{n}(x \mp a)}\right| \sim O\left(e^{-\operatorname{Im} \zeta_{n} r}\right), \quad r=|x|-a \rightarrow \infty .
$$


Therefore, to provide the required accuracy outside some area $D_{F}:|x|<b$, which includes the location of inhomogeneity ( $a<b, D_{\text {in }} \subset D_{F}$ ), one can keep only a limited number of $N$ terms in expansion (2) (all real and a few first complex wavenumbers $\zeta_{n}$ ).

In accordance with expansion (2), the stress vector $\sigma(\mathbf{x})=\left(\sigma_{x x}, \tau_{x z}\right)=(\sigma, \tau)$, which will be needed below for the boundary conditions on the waveguide's cross-section $x=$ const, is also represented in terms of GWs:

$$
\sigma_{\mathrm{sc}}(x, z)=\sum_{n=1}^{\infty}\left\{\begin{array}{cl}
c_{n}^{-} \mathbf{b}_{n}^{-}(z) e^{-i \zeta_{n}(x+a)}, & x<-a \\
c_{n}^{+} \mathbf{b}_{n}^{+}(z) e^{i \zeta_{n}(x-a)}, & x>a .
\end{array}\right.
$$

The normal mode eigenforms $\mathbf{b}_{n}^{ \pm}(z)$ are expressed via $\mathbf{a}_{n}^{ \pm}(z)$ in conformity with the stress component representation:

$$
\sigma_{x x}=\sigma=\lambda \operatorname{divu}+2 \mu \frac{\partial w}{\partial z}, \quad \tau_{x z}=\tau=\mu\left(\frac{\partial u}{\partial x}+\frac{\partial w}{\partial z}\right)
$$

The wavenumbers $\zeta_{n}$ are roots of the characteristic (dispersion) equation for the waveguide considered, and the eigenforms $\mathbf{a}_{n}$ are eigensolutions in the Fourier transform domain associated with these roots. Conventionally, they are calculated using the modal analysis technique, but we traditionally obtain them from the Green's matrix based integral representation of the force-generated solution in the intact waveguide [6]:

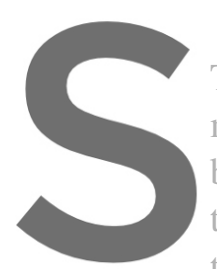

$$
\mathbf{u}=k * \mathbf{q}=\int_{\Omega} k(x-\xi, z) \mathbf{q}(\xi) d \xi=\frac{1}{2 \pi} \int_{\Gamma} K(\alpha, z) \mathbf{Q}(\alpha) e^{-i \alpha x} d \alpha .
$$

This equality gives the wavefield $\mathbf{u} \mathbf{x})$ generated by a surface load $q(x)$ as a contolution of the Green's
matrix $k$ and vector $q$ of, alternatively, in the form of inverse path Fourier integtal of their Fourier sym-
bols: $K(\alpha, z)=\mathcal{F}[k(x, z)]$ and $\mathbf{Q}(\alpha)=\mathcal{F}[\mathbf{q}(x)] ; \mathcal{F}$ is the Fourier transform operator with respect to
the $x$ variable. The roots $s_{n}$ coincides with the poles of the matrix $K$ elements. and the residues from
these poles to constant factors coimcide with the comresponding eigensolutions. Therefore, the Tesidue technique applied to the path integral in Eq. (3) reduces it to the sums of form (2), in which Register for free at https//www.scipedia.com to download the version without the watermark

$$
\mathbf{a}_{n}^{ \pm}=\left.\mp \operatorname{ires} K(\alpha, z) Q(\alpha)\right|_{\alpha=\mp \zeta_{n}} .
$$

This way of obtaining expansion (2) is more convenient for us because we have elaborated and computer implemented a series of fast and numerically stable algorithms of matrix $K$ calculation for multilayered, functionally graded, anisotropic, porous, cylindrical, and other elastic wavequides (e.g., [7-9]). Thus, the described below hybrid scheme FEM-An can be implemented for all such waveguides.

\subsection{General scheme}

Given representation (2), a general solution in the entire infinite domain can be sought in the following form

$$
\mathbf{u}(\mathbf{x}) \approx \mathbf{u}_{N}(\mathbf{x})=\left\{\begin{array}{lr}
\mathbf{u}_{0}(\mathbf{x})+\mathbf{u}_{\mathrm{sc}, N}^{-}(\mathbf{x}), & x \leq-b \\
\mathbf{u}_{F}(\mathbf{x}), & |x| \leq b \\
\mathbf{u}_{\mathrm{sc}, N}^{+}(\mathbf{x}), & x \geq b .
\end{array}\right.
$$


Here $\mathbf{u}_{0}$ is an incident wavefield, $\mathbf{u}_{s c, N}^{ \pm}$are first $N$ terms of expansion (2), and $\mathbf{u}_{F}=\left(u_{F}, w_{F}\right)$ is a FEM solution in the local area $D_{F}$. For definiteness, we assume that the incident wave $\mathbf{u}_{0}$ comes from the left; if the source is located in $D_{F}$, then $\mathbf{u}_{0} \equiv 0$. The number of terms $N$ and the borders $x= \pm b$ should be chosen so that the contribution of the discarded terms does not exceed a certain specified error $\varepsilon$. The stress field $\sigma(\mathbf{x}) \approx \sigma_{N}(\mathbf{x})$ is of the same structure as $\mathbf{u}_{N}$ with representation (3) for $\sigma_{s c, N}^{ \pm}$in the exterior domains $D^{ \pm}$and a FEM obtained field $\sigma_{F}=\left(\sigma_{F}, \tau_{F}\right)$ in $D_{F}$.

The artificial boundary $x= \pm b$ should not spoil the continuity of the displacement and stress vectors $\mathbf{u}(\mathbf{x})$ and $\sigma(\mathbf{x})$. That is, the equalities

$$
\begin{aligned}
\left.\left(\mathbf{u}_{0}+\mathbf{u}_{s c}^{-}\right)\right|_{x=-b}=\left.\mathbf{u}_{F}\right|_{x=-b}, & \left.\mathbf{u}_{F}\right|_{x=b}=\left.\mathbf{u}_{s c}^{+}\right|_{x=b} \\
\left.\left(\sigma_{0}+\sigma_{s c}^{-}\right)\right|_{x=-b}=\left.\sigma_{F}\right|_{x=-b}, & \left.\sigma_{F}\right|_{x=b}=\left.\sigma_{s c}^{+}\right|_{x=b},
\end{aligned}
$$

must be satisfied. However, the scattered fields $\mathbf{u}_{s c}^{ \pm}$and $\sigma_{s c}^{ \pm}$are determined up to unknown coefficients $c_{n}^{ \pm}$. Therefore, it is not possible to compute and set the arrays of input data at the boundaries, which are required for the FEM packages to work. To use FEM, the values $c_{n}^{ \pm}$have to be already obtained, which is impossible before the values of $\mathbf{u}_{F}$ and $\sigma_{F}$ are calculated. In addition, the FEM computation requires to specify only two functions on the border $\partial D_{F}$ out of four components of the vector-functions $\mathbf{u}_{s c}$ and $\sigma_{s c}$ that appear in conditions (5) and (6). That is, the FEM problem is overdetermined, and to form the input data, one can only use either conditions (5) or (6).

To avoid these difficulties, we have formulated $2 N+1$ auxiliary FEM problems with the boundary conditions induced by $\mathbf{u}_{0}$

taken in the form of linear coefficients $c_{n}^{ \pm}$:
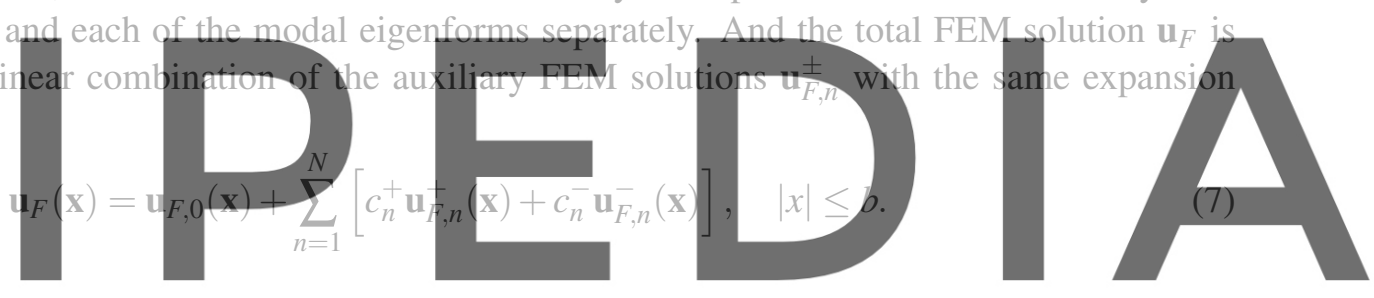

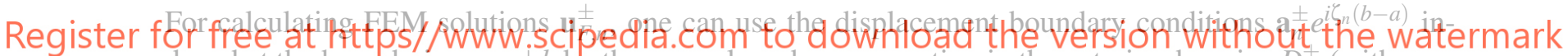
duced at the boundaries $x= \pm b$ by the normal modes propagating in the exterior domains $D^{ \pm}$(with zero

displacements at the opposite end). In such a case, conditions (5) of displacement continuity are satisfied automatically, and the total field $\mathbf{u}_{N}(\mathbf{x})$ visually looks smooth. However, it cannot correctly meet the condition of wave energy radiation to infinity because the Umov-Poynting vector, specifying time-averaged wave energy transfer in a time-harmonic field, include both displacement and stress components. To keep energy balance, the stress field must be also continuous. To meet conditions (6) of stress continuity, one can use the remaining $2 N$ degrees of freedom provided by the coefficients $c_{n}^{ \pm}$. The discretization of these boundary conditions can be performed in various ways, e.g., by equating at $N$ nodes on each side or using the Galerkin method. In the latter, it is advantageous to take components of the same normal modes $\mathbf{a}_{n}^{ \pm}$and $\mathbf{b}_{n}^{ \pm}$as projectors and use the property of normal modes' orthogonality [10]:

$$
\left(a_{m, x}, b_{n, x}\right)-\left(b_{m, z}, a_{n, z}\right)=0, \quad m \neq n, \quad \text { where } \quad(f, g)=\int_{-H}^{0} f(z) g(z) d z .
$$

To use orthogonality, the FEM problems should be with mixed boundary conditions (one displacement component and one stress component, say $\sigma_{F, n}=b_{n, x}$ and $w_{F, n}=a_{n, z}$ at one end and zero at the other). 
Such discretization reduces the problem to a linear algebraic system with respect to the vector of unknown coefficients $\mathbf{c}=\left(c_{1}^{-}, c_{2}^{-}, \ldots, c_{N}^{-}, c_{1}^{+}, c_{2}^{+}, \ldots, c_{n}^{+}\right)$. The orthogonality provides a diagonal matrixblock $D$ in the system's matrix $A$, which makes it well-conditioned:

$$
\begin{gathered}
A \mathbf{c}=\mathbf{f} . \\
A=B+D, \quad \mathbf{f}=\mathbf{f}_{0}+\mathbf{f}_{F}, \\
B=\left[\begin{array}{cc}
B_{1}^{-} & B_{1}^{+} \\
B_{2}^{-} & B_{2}^{+}
\end{array}\right], \quad \begin{array}{r}
b_{1, n m}^{ \pm}=\left(u_{F, m}^{ \pm}, b_{n, x}^{-}\right)-\left(\tau_{F, m}^{ \pm}, a_{n, z}^{-}\right), \quad x=-b, \\
b_{2, n m}^{ \pm}=\left(u_{F, m}^{ \pm}, b_{n, x}^{+}\right)-\left(\tau_{F, m}^{ \pm}, a_{n, z}^{+}\right), \quad x=b .
\end{array} \\
D=\operatorname{diag}\left(d_{1}^{-}, d_{2}^{-}, \ldots, d_{N}^{-}, d_{1}^{+}, d_{2}^{+}, \ldots, d_{N}^{+}\right), \quad d_{m}^{ \pm}=-\left[\left(a_{m, x}^{ \pm}, b_{m, x}^{ \pm}\right)-\left(b_{m, z}^{ \pm}, a_{m, z}^{ \pm}\right)\right] e^{i \zeta_{m} r}
\end{gathered}
$$

Vector $\mathbf{f}_{0}$ is almost a null vector except for the component $f_{0, m}=\left[\left(a_{m, x}^{+}, b_{m, x}^{-}\right)-\left(a_{m, z}^{-}, b_{m, z}^{+}\right)\right] e^{i \zeta_{m} r}$, where $m: \zeta_{m}=\zeta_{0}$ in the incident wave $\mathbf{u}_{0}$. The components of vector $\mathbf{f}_{F}=\left(f_{F .1}^{-}, \ldots, f_{F, N}^{-}, f_{F .1}^{+}, \ldots, f_{F, N}^{+}\right)$are $f_{F, n}^{ \pm}=\left(u_{F, 0}, b_{n, x}^{ \pm}\right)-\left(\tau_{F, 0}, a_{n, z}^{ \pm}\right)$at $x= \pm b$.

\section{NUMERICAL EXAMPLES}

\subsection{FEM-An verification}

Applicability of the FEM-An scheme was first demonstrated with an analytically solvable test case [6].

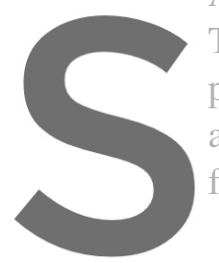

The waveguide was taken

patch bonded to the surface

and normal stresses $\left(\sigma_{z}\right.$

free except a point $\mathbf{x}_{0}=$
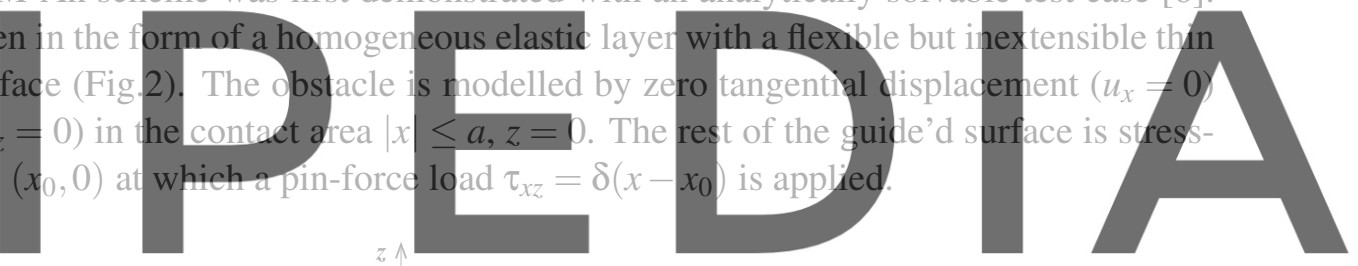

Register for free at https//www.șcipedia.com to ${ }^{\prime}$ dowrflozd the version without the watermark

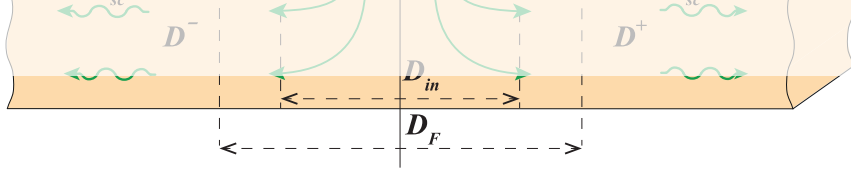

Figure 2: Geometry of test examples.

This is a classical elastic waveguide, which supports the antisymmetric and symmetric Lamb waves $A_{n}$ and $S_{n}$. The real and complex branches $\operatorname{Re} \zeta_{n}(\omega)$ and $\operatorname{Im} \zeta_{n}(\omega)$ of several first Lamb modes are shown in Fig. 3. Here, the dimensionless wavenumbers $\zeta_{n}$ are functions of the dimensionless angular frequency $\omega=2 \pi f H / c_{s}$, where $f$ is dimensional frequency, $H$ is the guide's thickness, and $c_{s}$ is the shear-wave velocity in the elastic material. This dimensionless form is based on the three basis units: length $l_{0}=H$, velocity $c_{0}=c_{s}$, and density $\rho_{0}=\rho$. It is also used in the examples of subsection 3.2 below.

The geometry of the test problem allows reducing it to the Wiener-Hopf integral equation for the unknown contact stresses $\tau_{x z}=q(x),|x| \leq a, z=0$. The right-hand side of this equation $f(x)=-u_{0}(x, 0)$. 


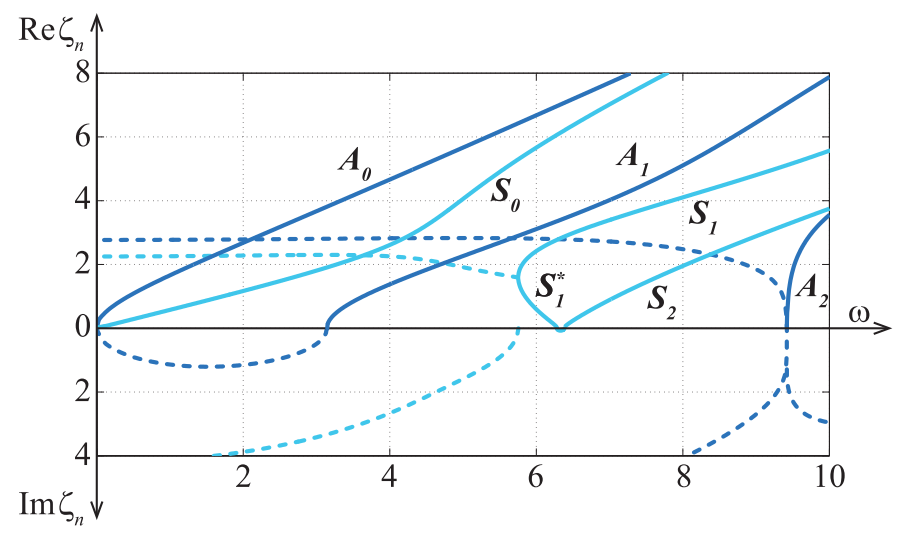

Figure 3: Real (solid lines) and imaginary (dashed lines) parts of the dimensionless Lamb wave dispersion curves; Poisson's ratio $v=0.34$.

The solution to such an integral equation can be obtained semi-analytically by the method of factorization [11] or using the residue technique that reduces it to a system of linear algebraic equations [13].

Thus, it made it possible to compare the three approaches:

a) by solving integral equation ("exact" solution Int-Eq);

b) by using standard FEM package with PML absorbing conditions (FEM-PML);

c) by using the hybrid scheme FEM-An.

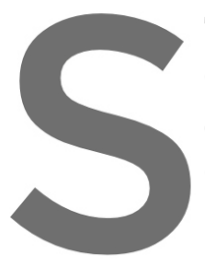

The characteristic feature

GWs with the opposite

dispersion curve with a neg

6.28 , and the beginning

show good agreement
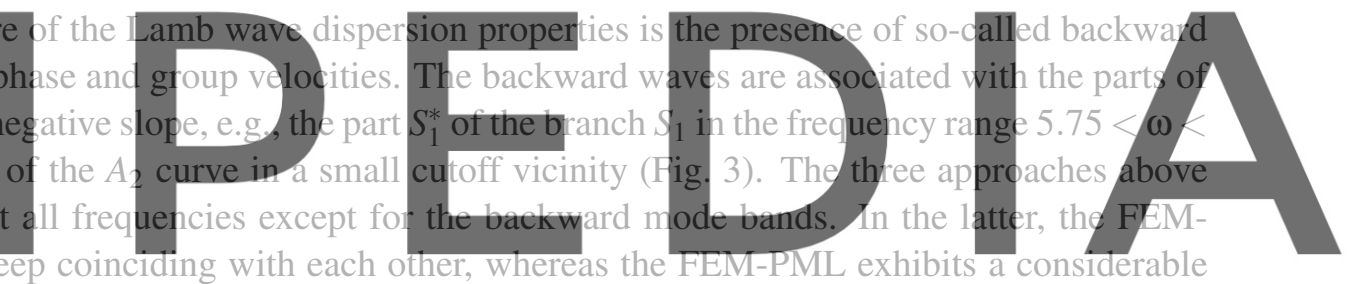

An and Int-Eq results keep coinciding with each other, whereas the FEM-PML exhibits a considerable
deviation. This is illustrated by the example of frequency dependence of the transmission coefficient

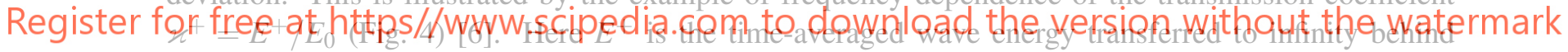

the obstacle by the field $\mathbf{u}_{s c}^{+}$, and $E_{0}$ is the total time-averaged power supplied by the point source at

$x_{0}=-5$; the obstacie is of hail-width $a=1$, and the hali-width of the FEII area $D_{F}$ is $b=10$ for FEII-

PML and $b=4$ for FEM-An. Since the Int-Eq results are exact, the FEM-PML deviations indicate the inapplicability of standard FEM packages to infinite waveguide at the backward-mode ranges, even if it is reduced to a finite domain by correct absorbing boundary conditions.

As an additional example aimed to show that the FEM-PML method fails at the backward mode frequencies not only for the wave energy transfer through the area of inhomogeneity, Figs. $5-6$ display numerical results for the case when the surface patch works as a piezoelectric actuator (PWAS [12]). Under a vertically polarised time-harmonic driven electric field, the bonded to the surface piezoelectric patch stretches and contracts generating GWs. Figure 5 depicts the frequency dependence of the total wave power $E_{0}$ supplied into the guide by this source with a constant driven voltage and carried to infinity by the traveling GWs. These dimensional results are obtained for an aluminium plate of thickness $H=1$ $\mathrm{mm}, c_{s}=3132 \mathrm{~m} / \mathrm{s}, \rho=2700 \mathrm{~kg} / \mathrm{m}^{3}$, and $\mathrm{v}=0.34$. The dimensionless dispersion curves of Fig. 3 are applicable in this case with the frequency unit (multiplier) $f_{0}=c_{s} / H=3.132 \mathrm{MHz}$. The range $0 \leq f \leq 4$ 


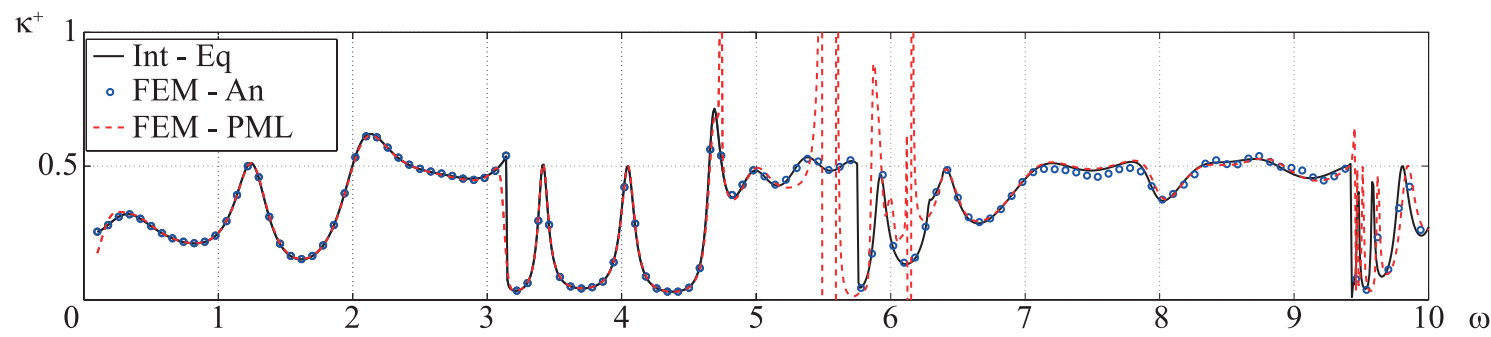

Figure 4: Transmission coefficient $\varkappa^{+}(\omega)$ : exact solution Int-Eq (solid line), FEM-PML (dashed line), and FEMAn (markers).

MHz of Fig. 5 corresponds to the dimensionless range $0 \leq \omega \leq 8.025$ of Fig. 3, and the backward mode band is $2.87<f<3.13(\mathrm{MHz})$.

Indeed, one can see that, at these frequencies, the FEM-PML dashed line is very different from the FEM-An solid line. Besides, unlike Fig. 4, the differences are also noticeable at some other frequencies. Apparently, the half-width $b=6 \mathrm{~mm}$ selected for the FEM area $D_{F}$ in both approaches was not large enough to proper simulate the infinite guide by the FEM-PML method. At the same time, the FEM-An is insensitive to the $D_{F}$ size if a sufficient number of modes $N$ is retained in the fields $\mathbf{u}_{s c}^{ \pm}$.

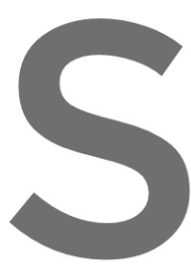

E, $\mu \mathrm{J}$
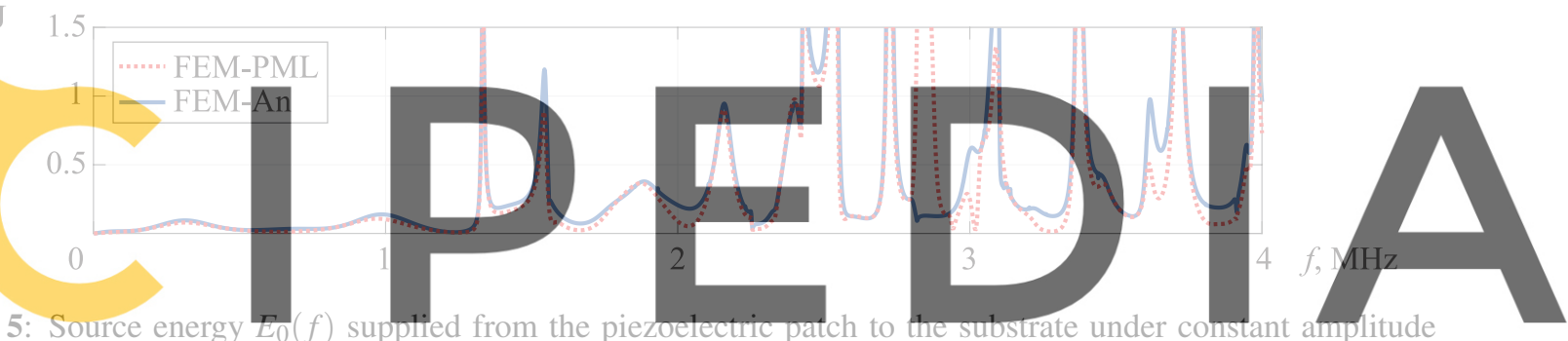

voltage calculated by FEM-An (solid line) and FEM-PML (dashed line),

Register for free at https//www.scipedia.com to download the version without the watermark

The FEM-PML inapplicability at the backward-mode frequencies affects not only the description of energy transfer but also other wave field characteristics. For example, the patterns of oscillation amplitude $|\mathbf{u}(x, z)|$ under the piezopatch coincide at a regular frequency (Fig. 6a,c) and visually differ at the backward-mode range (Fig. 6b,d).

\subsection{Multiple inclusions}

With its increased accuracy compared to standard FEM and significantly lower computational expenses, the FEM-An is a useful tool for fast parametric analysis of wave phenomena in elastic structures with complicated local obstacles. The next numerical example is for an elastic plate with a set of equidistant inclusions (Fig. 7). With an infinite number of such identical obstacles, the structure becomes a phononic crystal featured by pass and stop (or gap) bands. In the former, a traveling wave $\mathbf{u}_{0}$ passes without reflection, and in the latter, it is completely blocked . The FEM-An-based parametric analysis allows monitoring the pass and gap bands' formation as the number of obstacles $N$ increases.

The plots of the transmission coefficients $\varkappa^{+}(\omega)=E^{+} / E_{0}$ in Fig. 8 illustrate such a formation of the 

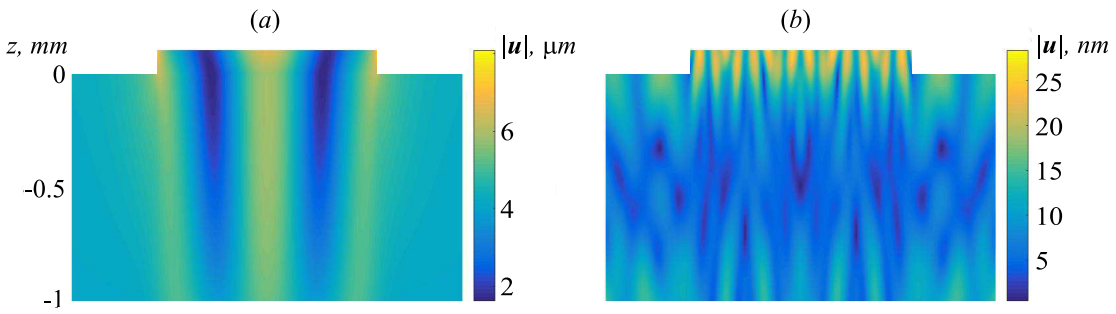

(c)
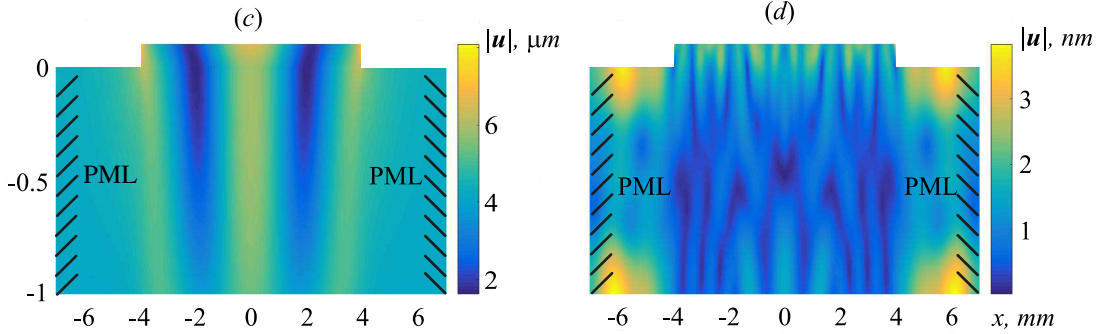

Figure 6: Displacement amplitudes $|\mathbf{u}(x, z)|$ under the piezopatch actuator: at the regular frequency $f=0.15 \mathrm{MHz}$ (left, (a), (c)), and the backward mode frequency $f=2.9 \mathrm{MHz}$ (right, (b), (d)); FEM-An - top (a), (b), FEM-PML - bottom (c), (d).
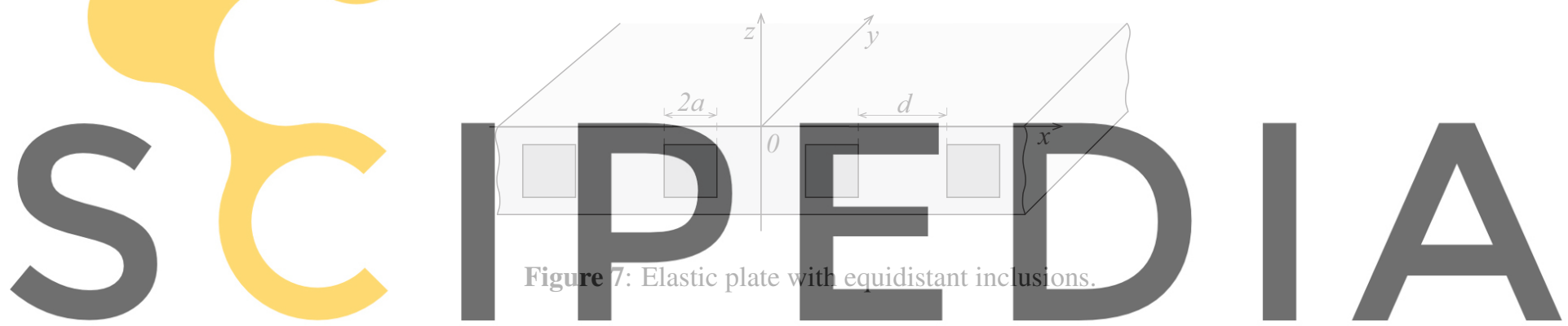

pass and gap frequency ranges for the $A_{0}$ and $S_{0}$ incidence (left and right subplots, respectively). The

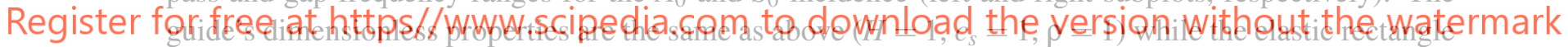

inclusions are specified by the parameters: $v=0.3, c_{s}=2, \rho_{2}=1.57,2 a=0.6$, and $d=2 ; E_{0}$ is the total

energy carried by the incident wave $\overrightarrow{\mathbf{u}}_{0}$ through the guide's cross-section before the obstacles, and $E^{+}$ is its part remaining behind them. As expected, the pass and gap bands visually appear as $N$ increases, similarly as it was demonstrated with a simpler $1 D$ guide with periodic point-wise defects [14].

\subsection{Disbonds in laminate plates}

The examples above were for a homogeneous elastic layer. To demonstrate the FEM-An practical applicability to multilayered structures, let us consider a typical GW-SHM problem of delamination detection in laminate composite plates [12]. Their locating and size estimates are based on the study of GW diffraction by an interface crack (Fig. 9). As an example, Fig. 10 depicts the variation of the frequency pattern of the transmission coefficient $\varkappa^{+}(f)$ as the crack half-width $a$ varies from 2.5 to $7.5 \mathrm{~mm}$. The plate is composed of four sublayers of thickness $h=0.575 \mathrm{~mm}$ (the total thickness $H=2.3 \mathrm{~mm}$ ), two external and two inner sublayers are of the same material properties: $v_{1}=0.323, c_{s, 1}=1026 \mathrm{~m} / \mathrm{s}, \rho_{1}=1522 \mathrm{~kg} / \mathrm{m}^{3}$ and $v_{2}=0.247, c_{s, 2}=2514 \mathrm{~m} / \mathrm{s}, \rho_{2}=1522 \mathrm{~kg} / \mathrm{m}^{3}$ for the former and latter, respectively. The crack is 

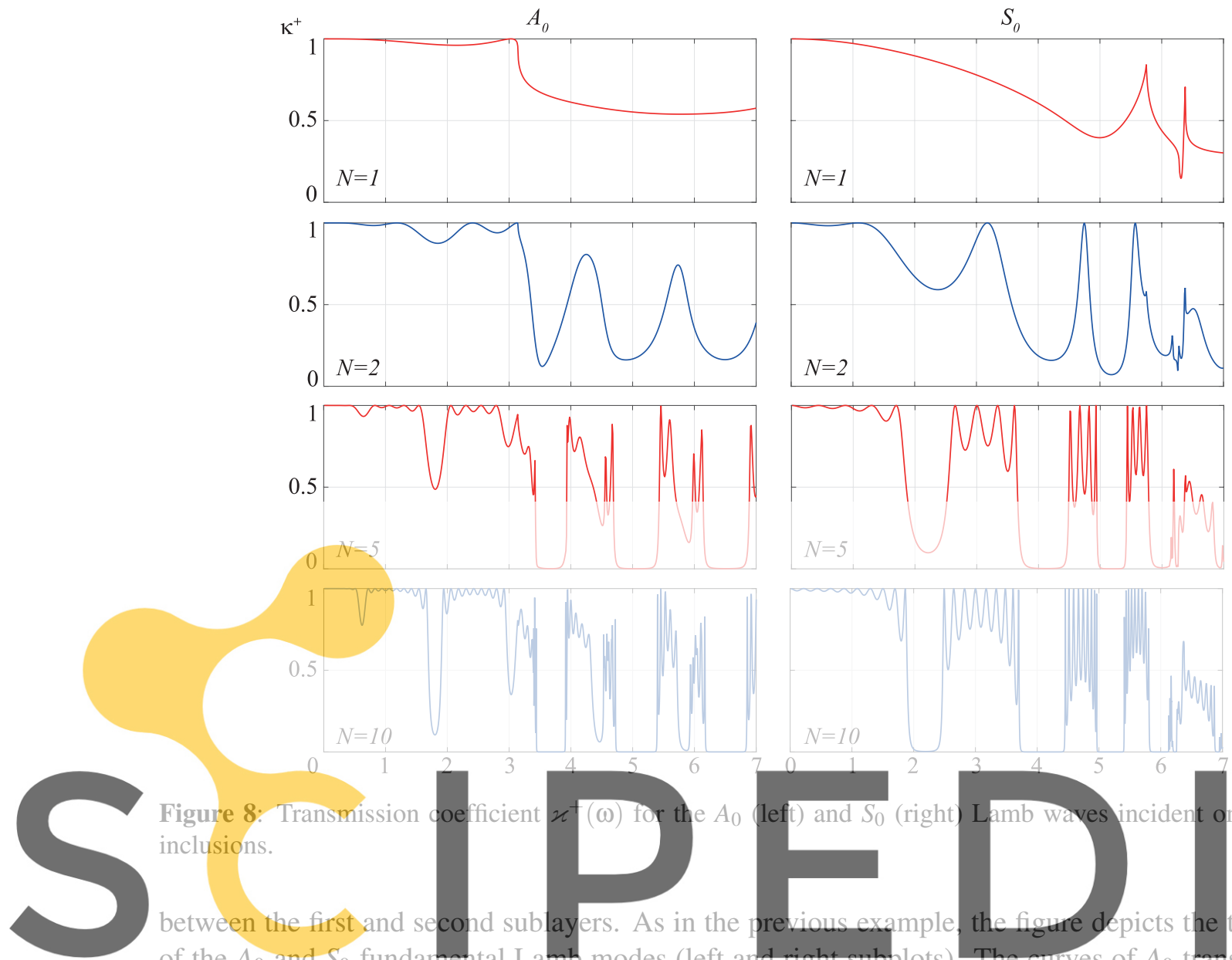

Figure 8: Transmission doefficient 2 inclusions.

between the first and second sublayers. As in the pret of the $A_{0}$ and $S_{0}$ fundarnental Lamb modes (left and
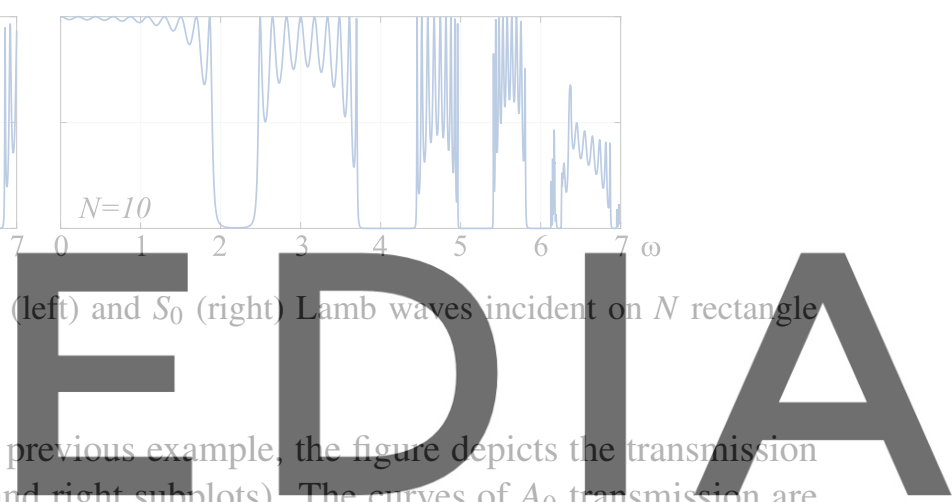

featured by sharp dips indicating abrupt blocking of $A_{0}$ propagation by the crack at specific resonance

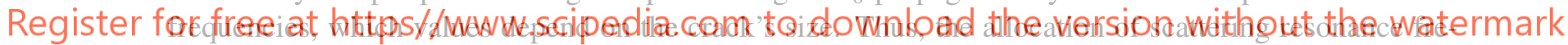
quencies extracted from the experimentally obtained GW frequency response can serve as additional information to estimate delamination severity. With the $S_{0}$ incidence (Fig. 10, right), the resonance dips and peaks are not very visible. Nevertheless, to estimate the crack size, one can use the range of $S_{0}$ full passing $0<f<f_{0}$, which shrinks from $f_{0} \approx 200$ to $f_{0} \approx 75(\mathrm{kHz})$ as the half-width $a$ increases from 2.5 to $7.5(\mathrm{~mm})$.

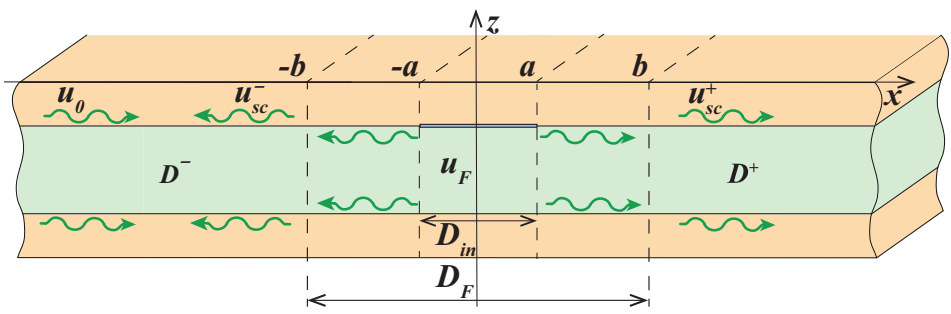

Figure 9: Multilayered structure with an interface delamination. 

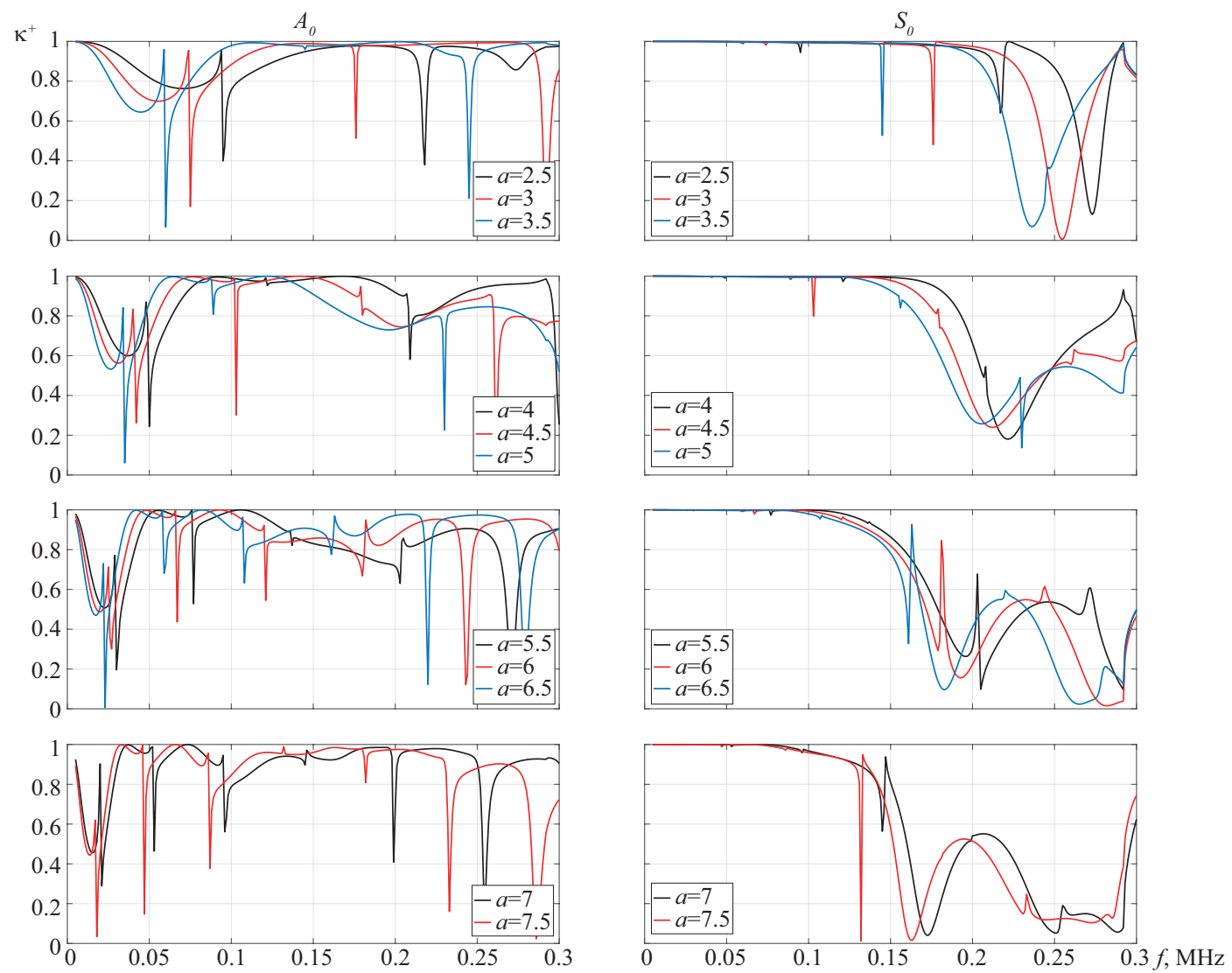

Figure 10: The change of frequency patterns of the transmission coefficient $\varkappa(f)$ for the $A_{0}$ (left) and $S_{0}$ (right) guided wave incident on the delamination of varying half-width $a$.

\section{CONCLUSION}

The main idea of the local-global numerical-analytical scheme FEM-An is described, verified, and illustrated by numerical examples for multiple obstacles and multilayered waveguide structures.

\section{REFERENCES}

[1] Shen, Y. and Giurgiutiu, V. Effective non-reflective boundary for Lamb waves: Theory, finite element implementation, and applications. Wave Motion. (2015) 58:22-41.

[2] Berenger, J.-P. A perfectly matched layer for the absorption of electromagnetic waves. J. Computational Physics. (1994) 114(2):185-200.

[3] Moulin, E., Assaad, J., Delebarre, Ch. and Osmont, D. Modeling of Lamb waves generated by integrated transducers in composite plates using a coupled finite element-normal modes expansion method. J. Acoust. Soc. Am. (2000) 107(1):87-94.

[4] Pinto, T. and Coyette, J.-P. Conjugated infinite elements for two-dimensional time-harmonic elastodynamics. J. Computational Acoustics. (2000) 8(3):473-493. 
[5] Gravenkamp, H., Birk, C. and Song, Ch. Simulation of elastic guided waves interacting with defects in arbitrarily long structures using the Scaled Boundary Finite Element Method. J. Computational Physics. (2015) 295(15):438-455.

[6] Glushkov, E., Glushkova, N. and Evdokimov, A. Hybrid Numerical-Analytical Scheme for Calculating Elastic Wave Diffraction in Locally Inhomogeneous Waveguides. Acoustical Physics. (2018) 64(1):3-12.

[7] Glushkov, E., Glushkova, N., Fomenko, S. and Zhang, C. Surface waves in materials with functionally gradient coatings. Acoustical Physics. (2012) 58(3):339-353.

[8] Glushkov, E., Glushkova, N. and Eremin A. Forced wave propagation and energy distribution in anisotropic laminate composites. J. Acoust. Soc. Am. (2011) 129(5):2923-2934.

[9] Glushkov, E., Glushkova, N. and Fomenko S. Wave generation and source energy distribution in cylindrical fluid-filled waveguide structures. Wave Motion. (2017) 72:70-86.

[10] Zilbergleit, A. and Nuller, B. Generalized orthogonality of the homogeneous solutions to the dynamic problems of elasticity. Doklady Physics. (1977) 234(2):333-335.

[11] Vorovich, I. and Babeshko, V. Dynamic Mixed Problems of Elasticity for Non-classical Domains. Nauka Moscow, (1979) (in Russian).

[12] Giurgiutiu, V. Structural Health Monitoring with Piezoelectric Wafer Active Sensors. Elsevier Academic Press, 2nd ed., (2014).

[13] Glushkov, E., Glushkova, N., Kvasha, O. and Seemann, W. Integral equation based modeling of the interaction between piezoelectric patch actuators and an elastic substrate. Smart Mater. Struct. (2007) 16:650-664.

[14] Glushkov, E., Glushkova, N. and Wauer, J. Wave propagation in an elastically supported string with point-wise defects: gap-band and pass-band effects. Journal of Applied Mathematics and Mechanics. (2011) 91(1):4-22. 